\title{
Dwarf spheroidal galaxies in nearby groups imaged with HST
}

\author{
I.D. Karachentsev ${ }^{1}$, V.E. Karachentseva ${ }^{2}$ \\ and M.E. Sharina ${ }^{1}$ \\ ${ }^{1}$ Special Astrophysical Observatory, Russian Academy of Sciences, N.Arkhyz, Russia \\ email:ikar@sao.ru \\ ${ }^{2}$ Astronomical Observatory of Kiev University, Kiev, Ukraine
}

\begin{abstract}
An all-sky list of 88 nearby dwarf spheroidal (dSph) galaxies with distances $D<10$ $\mathrm{Mpc}$ is considered. Most of the objects have recently been found by Karachentseva \& Karachentsev based on POSSII/ESO-SERC survey. A hundred more dSph galaxies are expected in this volume, being missed so far because of their low luminosity and low surface brightness. Apart from $22 \mathrm{dSph}$ members of the Local Group, there are $33 \mathrm{dSphs}$ in other nearby groups that have been resolved recently into stars with HST. Only $5 \%$ of the local dSphs are situated outside the known groups.

We discuss observational correlation between basic parameters of the dwarf spheroidal galaxies, in particular, absolute magnitude, surface brightness, metallicity, and so-called "tidal index". The observed number of dSphs in group increases with luminosity of its brightest galaxy. In a "synthetic" nearby group, dwarf spheroidal galaxies are distributed in depth quite symmetrically about the principal galaxy, having an rms distance scatter of $200 \mathrm{kpc}$. Projected radial distribution of dSphs in the synthetic group follows the profile $N(R) \sim \exp (-R / 200 \mathrm{kpc})$.
\end{abstract}

Keywords. dwarf, fundamental parameters

\section{Introduction}

According to the entire sky catalog of 1500 low surface brightness dwarf galaxies with angular diameters larger than $0.4^{\prime}$ (Karachentseva \& Sharina 1988), dwarf spheroidal galaxies (dSphs) are strongly concentrated towards the nearest groups and clusters. Distributions of dSphs in Virgo and Fornax cluster were studied in details by Binggeli et al. 1987 and Ferguson \& Sandage 1989. A comprehensive review of basic properties of dwarf spheroidal galaxies in the Local Group was outlined by Mateo 1998. Recent mass measurements of accurate distances to nearby galaxies summarized in Catalog of 451 neighboring galaxies (Karachentsev et al. 2004) allow us to study distribution and properties of dSphs in the Local $(D<10 \mathrm{Mpc})$ Volume.

\section{DSph population within $10 \mathrm{Mpc}$}

Among 451 galaxies of the Local Volume (LV), 88 objects are classified as dSphs because of their smooth shape, low surface brightness, absence of $\mathrm{H}_{\alpha}$ emission and undetectably low HI flux. Nine of them (LGS-3, Phoenix, KK 27, KDG 61, Antlia, HS 117, DDO 113, KKR 25, and DDO 216) can be classified rather as dwarf galaxies of transition (dSph/dIr) type since the presence of a fraction of young stars or noticable HI content. Among 66 dSphs in the LV situated outside the Local Group, 50\% of the sample have being imaged so far with WFPC2 or ACS at Hubble Space Telescope. No nucleated galaxies have been found as yet. 


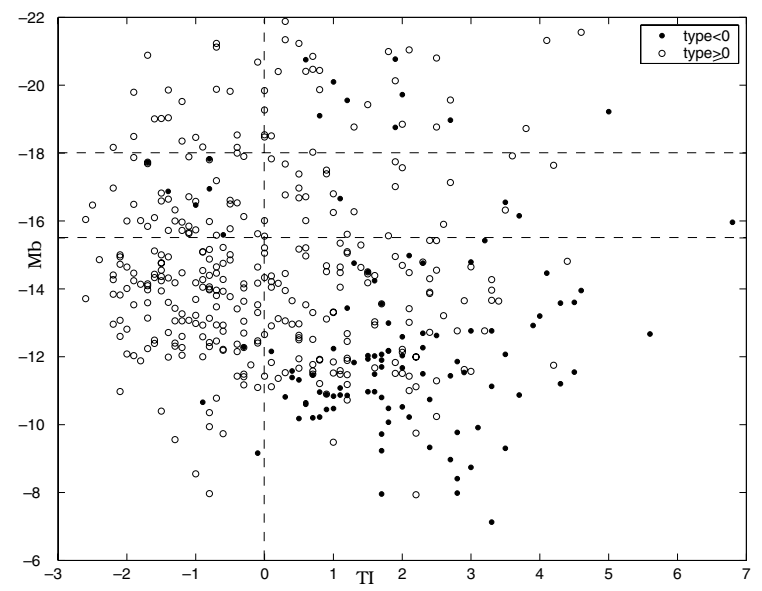

Figure 1. Absolute blue magnitude vs. tidal index for the LV galaxies

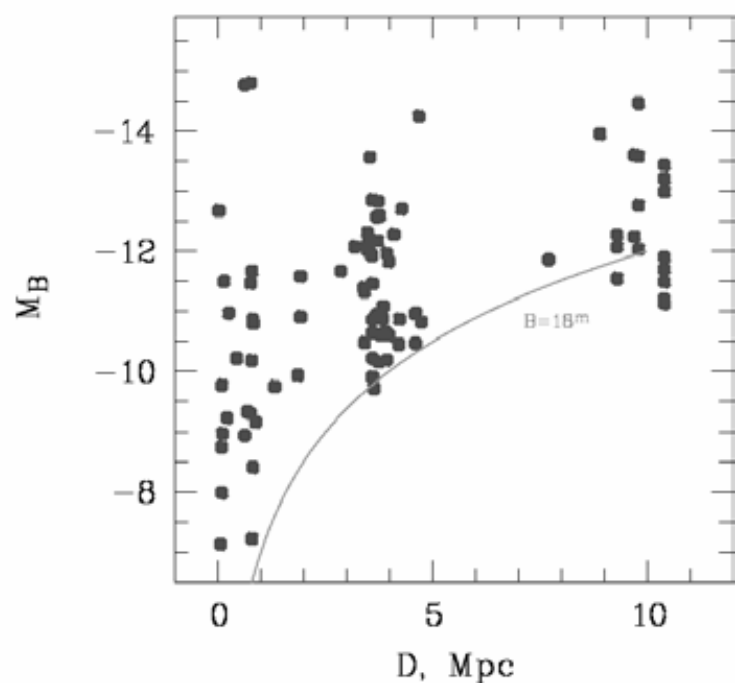

Figure 2. Absolute magnitude vs. distance for dSph galaxies

To quantify the environment of a LV galaxy, the so-called "tidal index" (or isolateness index) was used: $T I_{i}=\max \left[\log \left(M_{k} / D_{i k}^{3}\right]+C, \quad k=1,2, \ldots\right.$ where $M_{k}$ is the mass of a neighboring galaxy located at a distance $D_{i k}$ from the one considered; a constant $C$ is choosen so that the negative values of $T I$ correspond to isolated galaxies of the general field, while the positive values to group members. Fig. 1 shows the distribution of $451 \mathrm{LV}$ galaxies in blue absolute magnitude and tidal index. The filled circles indicate early-type objects $(T<0)$. Among the high-luminosity galaxies with $M_{B}<-18^{m}$, all $\mathrm{E}$ and SO galaxies are within the range of positive $T I$, i.e. in groups. On the bottom side, among dwarf galaxies with $M_{B}>-15.5^{m}$, the overwhelming majority of early- type (dSph) objects are in the dense environment $(T I>0)$ too.

Fig. 2 presents the distribution of dSph galaxies according to their distances and absolute blue magnitudes corrected for the Galactic extinction. The line corresponds to a limiting apparent magnitude $18^{m}$. Lack of the faintest galaxies towards the LV edge is clearly seen. Assuming our sample within $2 \mathrm{Mpc}$ to be $90 \%$ complete, we derive about $100 \mathrm{dSphs}$ missed within $10 \mathrm{Mpc}$ because of their faintness. 
The pair-wise distributions of the LV dSphs according to their absolute magnitude $M_{B}$, mean blue surface brightness $\Sigma_{B}$, apparent axial ratio $b / a$, and tidal index $T I$ are given in Fig. 3. Objects of the transition dSph/dIr type are indicated by triangles, and members of the Local Group are shown by filled symbols. As seen from these data, dwarf spheroidal galaxies outside the LG occupy about the same range of the considered parameters as the LG members themselves. There is no significant difference between galaxies of the dSph/dIr type and pure dSph objects either.
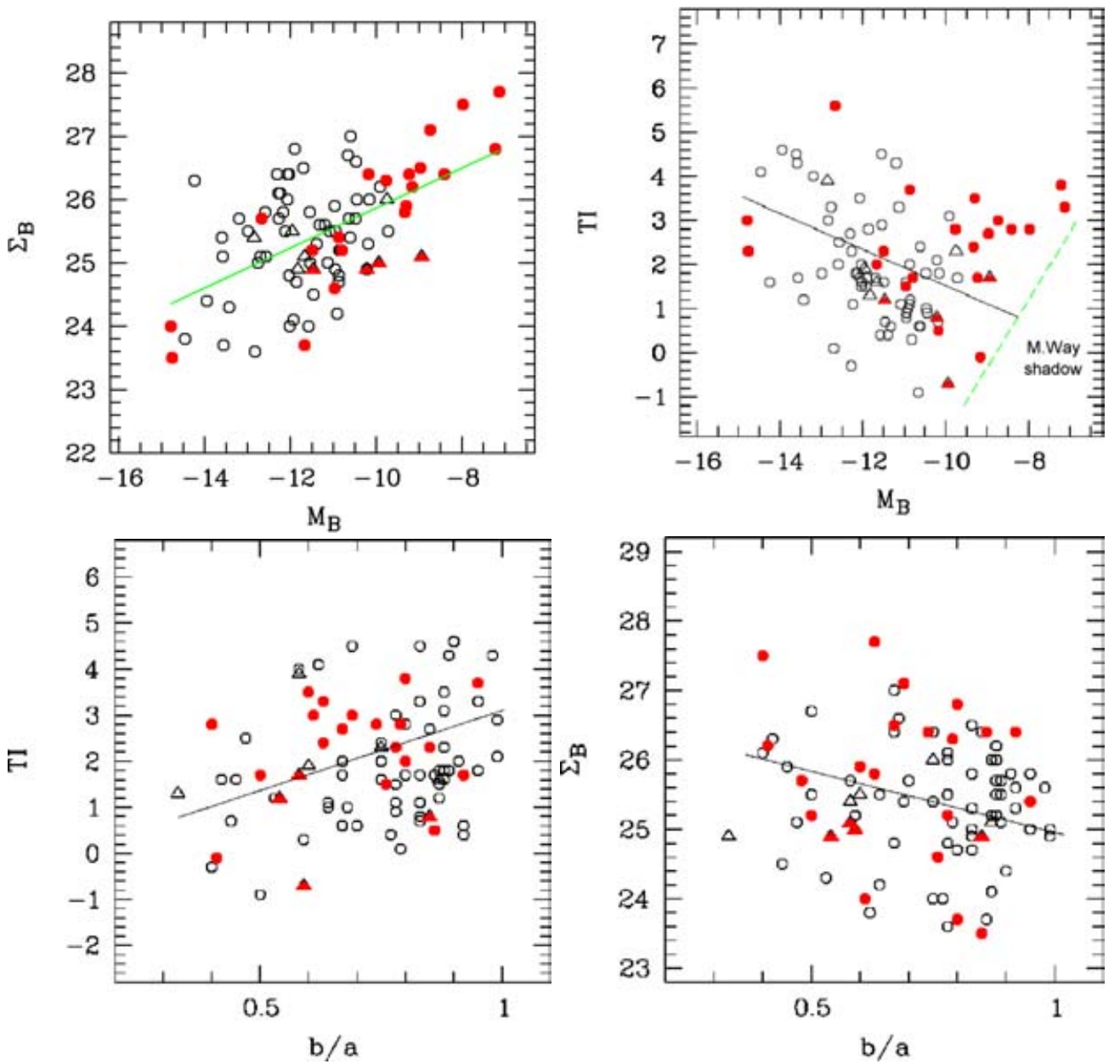

Figure 3. Correlations between absolute magnitude, average surface brightness, axial ratio and tidal index for dSphs. Galaxies of transition type are shown by triangles and members of the LG are indicated by filled symbols

\section{Dwarf spheroidal galaxies imaged with ACS HST}

Over the past few years, $33 \mathrm{dSph}$ galaxies outside the LG have been observed with WFPC2 and ACS at HST. This number exceeds the total number of dSphs in the Local Group itself $(\mathrm{N}=22)$. 600-second exposures were taken in the F606W and F814W filters for each object. The photometric reduction was carried out using the HSTphot stellar photometry package described by Dolphin 2000. The ACS I- images of the dSph galaxy 

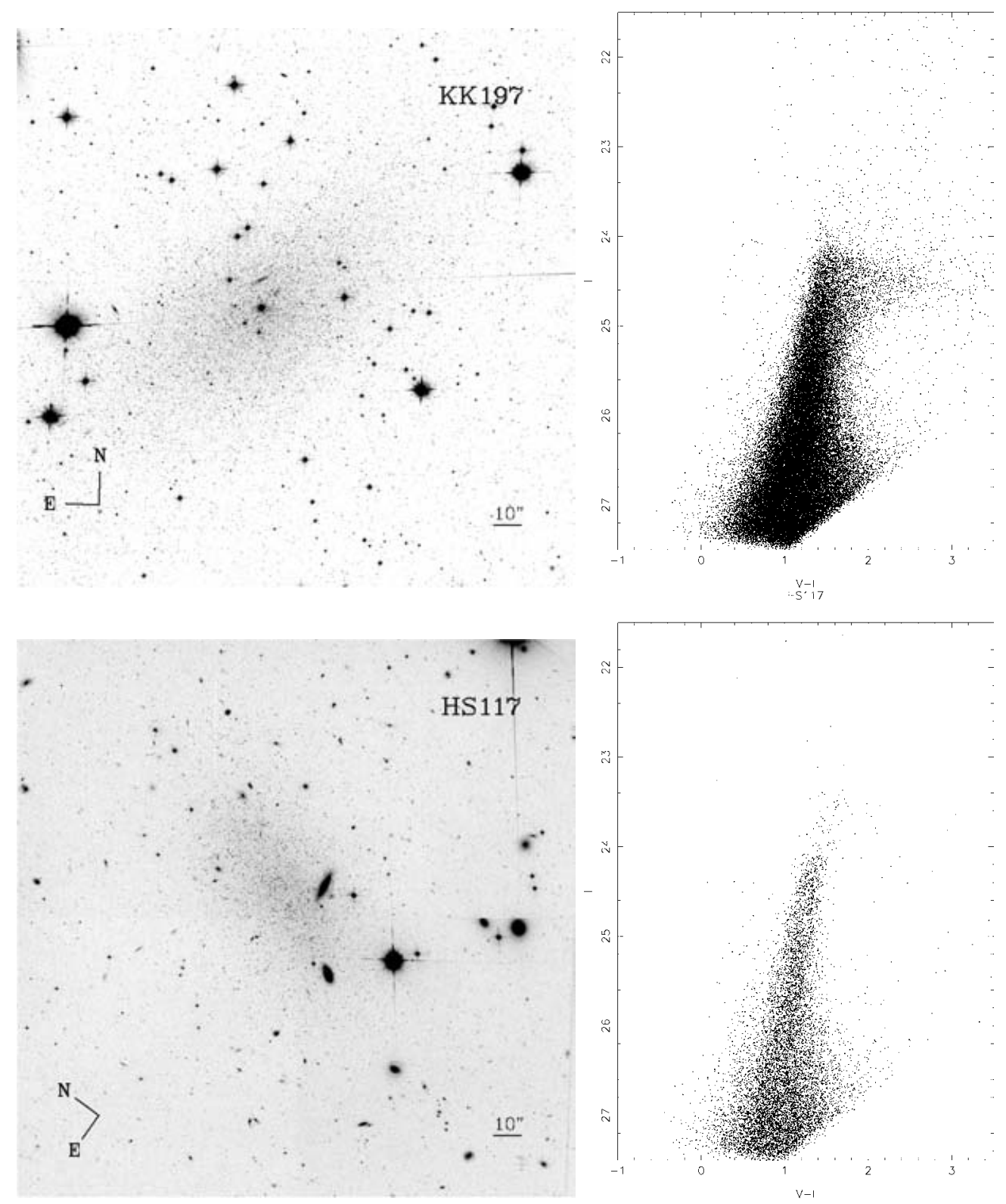

Figure 4. I-band ACS images and CM diagrams for dSph galaxy KK 197 and dSph/dIr galaxy HS 117

KK 197 in the Centaurus A group and the dSph/dIr galaxy HS117 in the M81 group are shown on left panel of Fig. 4. Their $(V-I)$ color-magnitude diagrams (right panel of Fig. 4) exhibit a lot of red giant branch (RGB) stars. Based on the tip of RGB, the galaxy distances are $3.73 \mathrm{Mpc}$ and $3.93 \mathrm{Mpc}$, respectively, confirming their membership in corresponding groups. The galaxy KK 197 has a blue absolute magnitude of -12.83 and a $50 \mathrm{kpc}$ projected separation from the giant galaxy Cen A. The galaxy HS 117 has a lower blue absolute magnitude -11.96 and a projected separation of $190 \mathrm{kpc}$ from the 
M81. The superior quality of ACS led to detection of about 44000 stars in KK 197 and about 9000 stars in HS 117.
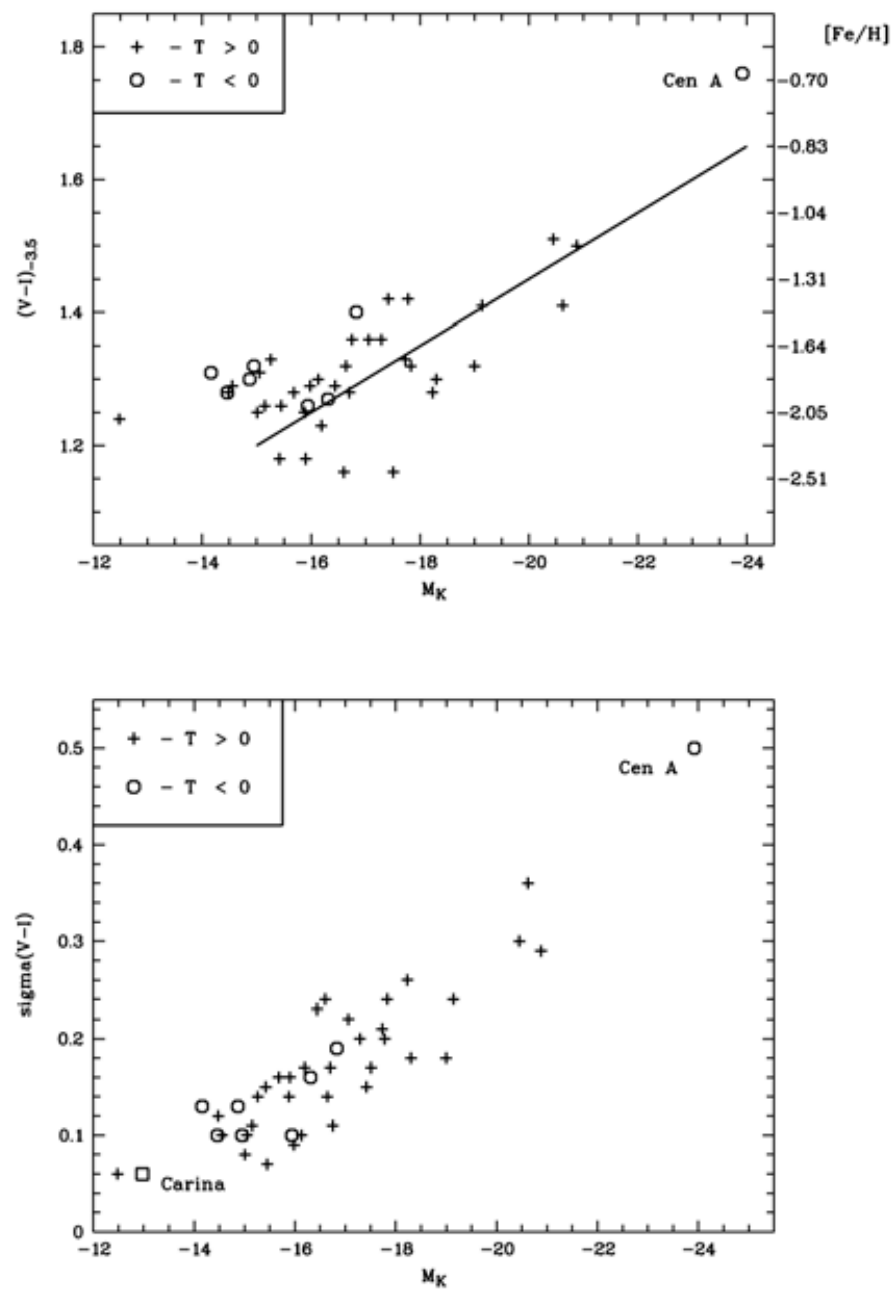

Figure 5. RGB color (upper panel) and dispersion of RGB colors vs K-band absolute magnitude for dSph (circles) and dIr (crosses) galaxies

\section{RGB color - luminosity relation}

As it was found by Da Costa \& Armandroff 1990 and Lee et al. 1993, the reddeningcorrected $(V-I)$ color of the RGB at $M_{I}=-3.5$ is a monotonic sequence with respect to galaxy metallicity $[\mathrm{Fe} / \mathrm{H}]$. According to Grebel et al. 2003, the metallicity of dwarf galaxies in the LG correlates with their luminosity, and dSphs are less luminous at equal metallicity than dIrs. We measured $(V-I)_{-3.5}$ colors for 42 nearby galaxies observed with ACS and compared them with the absolute magnitude of the galaxies in the Kband derived from 2MASS (Karachentsev \& Kut'kin 2005). A plot of the mean ( $V-$ $I)_{-3.5}$ color vs. absolute K-magnitude is shown on upper panel of Fig. 5. Late-type and 
early-type galaxies are indicated by crosses and open circles, respectively. We put on the plot also the giant elliptical galaxy Cen A taking its metallicity from Rejkuba 2004. Here, the right scale shows the metallicity conversed from $(V-I)$ color via the relation by Lee et al. 1993. The known color - luminosity relation, as well as the offset of dSphs with respect to dIrs is seen distinctly.

Apart from the mean RGB color, the scatter of RGB colors measured at the same $M_{I}=-3.5$ level exhibits a correlation with K-luminosity of the galaxies too (lower panel of Fig. 5). The existence of such a kind of relation can be suspected from the data on the LG galaxies collected by Mateo 1998. The LG galaxy Carina dSph having a dispersion of $[\mathrm{Fe} / \mathrm{H}]=0.25 \operatorname{dex}($ Koch et al. 2004) is indicated in Fig. 5 by open square. Surprisingly, we did not recognize any significant difference between dSphs and dIrs on the diagram.

\section{DSphs in a synthetic nearby group}

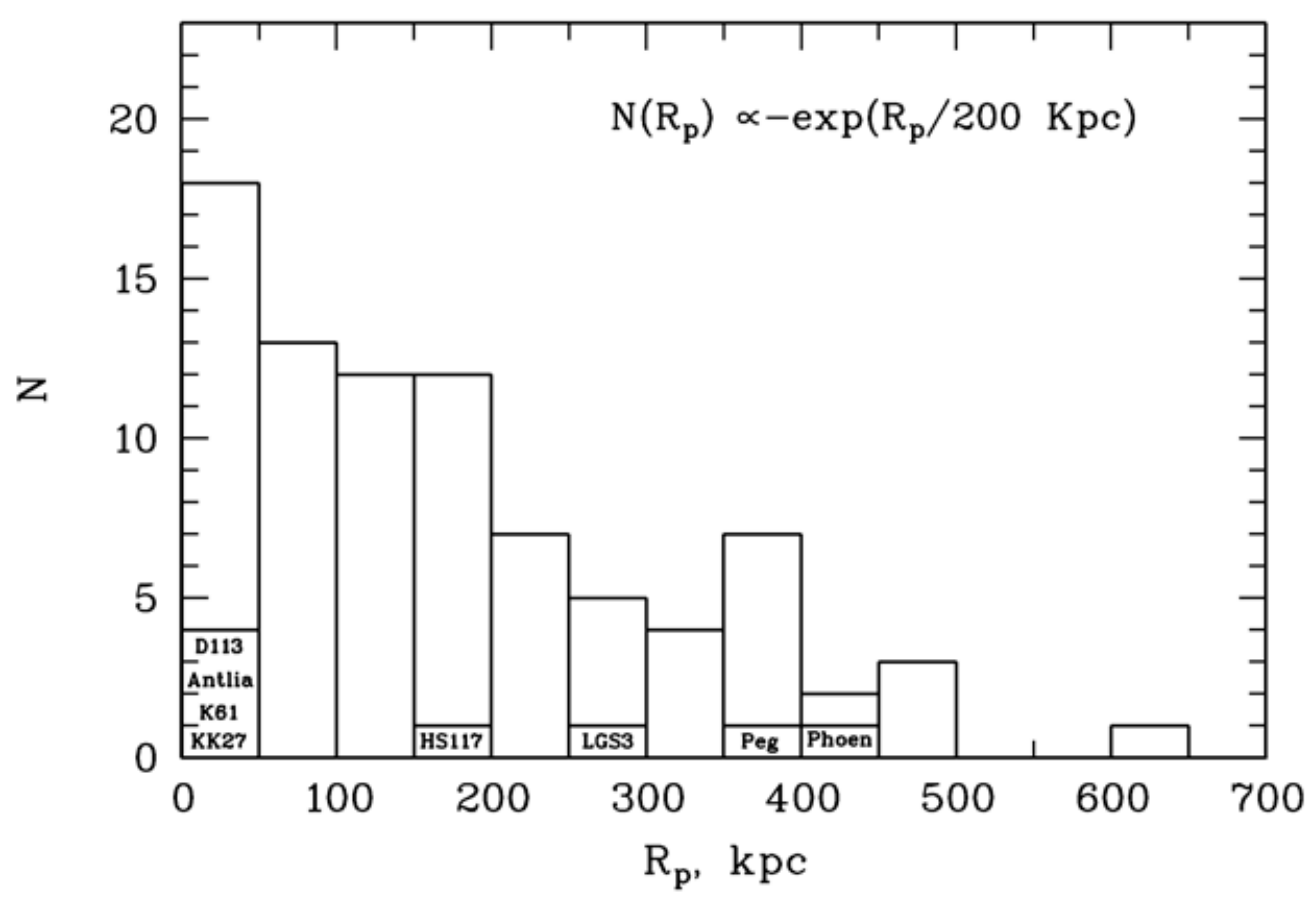

Figure 6. Number distribution of dSphs according to their projected distance from the brightest galaxy in nearby groups

Comparing numbers of dSph and dIr galaxies in nearby groups, Karachentsev 2005 noted that the fraction of dSphs tends to increase with luminosity of the bulge of the main group member and to decrease with crossing time of the group, which has a quite obvious evolutionary interpretation. Table 1 presents parameters of the brightest galaxy in nearby groups and numbers of $\mathrm{dSph}$ companions. Its columns give the following data: (1) name of the galaxies ranked according to their infrared luminosity, (2) morphological type of the brightest galaxy in each group, (3) mean distance to the group, (4) absolute K- magnitude of the main galaxy, (5) tidal index for the brightest galaxy (showing presence of another bright enough galaxy in the group), (6) total number of the known 
Table 1. Parameters of the brightest galaxy in a group and number of dSphs

\begin{tabular}{lrrlrrrrrrr}
\hline Main galaxy & $T_{1}$ & $\begin{array}{r}D \\
\mathrm{Mpc}\end{array}$ & $\left(M_{K}\right)_{1}(T I)_{1}$ & $N_{\text {tot }}$ & $N_{s p h}$ & $N_{s p h}^{c}$ & $\left\langle R_{p}\right\rangle_{s p h}$ \\
$\mathrm{kpc}$ & $\left\langle R_{p}\right\rangle_{I r}$ \\
$\mathrm{kpc}$
\end{tabular}

companions, (7) total number of dSph companions, (8) number of dSph companions brighter than $M_{B}=-11^{m}$ (this quantity reduces the incompleteness of distant groups with respect to nearby ones seen in Fig. 2), $(9,10)$ mean projected separations of the dSphs and of late-type companions from the principal galaxy.

These data show that 84 of $88 \mathrm{LV}$ dSphs are situated in the known groups. Among the rest four field objects, two (KKs3, KK 258) need to be still confirmed as true dwarf speroidal galaxies. The highest numbers of $\mathrm{dSphs}$ are seen around the most luminous giant galaxies of types $T<4$. The presence of another bright galaxy in group favours higher number of dSphs companions too (the case of M 81 and M 82). Note that the giant, but bulgeless galaxy M 101, which dominates in its luminosity over the rest of the group members, has no dSph companions at all. Comparing the mean projected separation of dSphs, $174 \mathrm{kpc}$, with the mean separation of the late-type companions, $276 \mathrm{kpc}$, we find that in the nearby groups dSphs occupy a volume, which is only a quater of the volume populated by the late-type members.

The distribution of dSphs according to their projected separations from the brightest group galaxy is presented in Fig. 6. Eight transition objects are indicated by their abbreviations. The observed distribution can be fitted by an exponent $N\left(R_{p}\right) \sim \exp \left(-R_{p} /\right.$ $200 \mathrm{kpc}$ ). No significant offset of dSph/dIr galaxies is seen with respect to the pure dSphs on this diagram. We also considered the distribution of distances of $39 \mathrm{dSph}$ companions along the line of sight with respect to the principal galaxy in nearby groups. The distribution looks to be quite symmetric showing the mean difference $\left.<D(d S p h)-D_{1}\right\rangle=$ $+24 \pm 33 \mathrm{kpc}$ and dispersion $202 \mathrm{kpc}$, which is roughly consistent with their distribution according to projected linear distances. 


\section{Brief conclusions}

We presented arguments that basic properties of dwarf spheroidal galaxies situated in nearby groups are quite similar to parameters of the Local Group dSphs that have been studied in much more details.

There are 88 known dSphs (and dSph/dIrs) within $10 \mathrm{Mpc}$, and a hundred dSphs are still missing in the same volume. Almost all dSphs in the LV ( 95\%) occur in galaxy groups. No "nucleated" galaxies fainter than $M_{B}=-15.5^{m}$ were found in the LV. This threshold corresponds (accidentally?!) to the "specific frequency" $=1$ for globular clusters.

Distributions of the LV dSphs according to their absolute magnitude, surface brightness, apparent axial ratio and tidal index show that a) on the $\left\{\Sigma_{B}\right.$ vs. $\left.M_{B}\right\}$ - diagram dSphs follow the line of constant spatial brightness, $\Sigma_{B} \sim(1 / 3) \times M_{B}$, b) the brighter $\mathrm{dSphs}$, the higher their tidal index is (survival effect?), c) round dSphs tend to be closer to giant galaxies, d) elongated dSphs tend to have lower $\Sigma_{B}$ (inclination effect?).

The RGB color - luminosity relation, known for the LG dSphs, is also valid for the dSphs in other nearby groups. The LV dSphs are found to be $\sim 1^{m}$ fainter than dIrs of the same metallicity, like dSphs in the Local Group. Surprisingly, the dispersion of metallicity (defined as the dispersion of RGB colors) decreases strongly ( 8 times!) from giant galaxies towards dwarfs.

The distribution of dSphs in nearby groups with respect to the principal galaxy has a characteristic scale of $200 \mathrm{kpc}$. The number of dSphs in group increases with bulge luminosity of the brightest group member. In the nearby groups, Sphs occupy only a quater of the volume populated by the late-type members of the groups.

\section{Acknowledgements}

This work was supported by RFFI grant 04-02-16115 and DFG-RFBR grant 02-0204012. This search has made use of the 2MASS survey.

\section{References}

Binggeli B., Tammann G.A. \& Sandage A. 1987, AJ 94, 251

Da Costa G.S. \& Armandroff T.E. 1990, AJ 100162

Dolphin A.E. 2000, ApJ 550, 554

Ferguson H.C. \& Sandage A. 1989, ApJ 346, 53

Karachentsev I.D. 2005, $A J$ 129, 178

Karachentsev I.D., Karachentseva V.E., Huchtmeier W.K. \& Makarov D.I. 2004 AJ 127, 2031

Karachentsev I.D. \& Kut'kin A.M 2005 Astronomy Letters 31, 299

Karachentseva V.E. \& Sharina M.E. 1988, Comm. Special Astrophys. Obs. 57, 5

Koch A., Grebel E.K., Harbeck D., et al. 2004 astro-ph/0410435

Lee M.G., Freedman W.L. \& Madore B.F. 1993, ApJ 417553

Mateo M. 1998,ARAA. 36, 435

Rejkuba M. 2004, astro-ph/0407636

\section{Discussion}

SHARINA: Can one make conclusions about metallicities of RGB stars without reliable transformations from the ACS instrumental to the standard Johnson-Cousins system available? The transformations are nonlinear and strongly depend on colour.

KARACHENTSEV: We made such conclusions using uniform data for all galaxies. Since there are no reliable transformations at the moment published, these conclusions are very preliminary. 